\title{
The stability of the ADO score among UK COPD patients from The Health Improvement Network
}

\author{
Spencer J. Keene (1) ${ }^{1,2,3}$, Peymane Adab (1) ${ }^{1}$, Frank de Vries (1) ${ }^{2,4,5}$, \\ Frits M.E. Franssen $\mathbb{1}^{3,6}$, Alice Sitch (10 ${ }^{1}$, James Martin ${ }^{1}$, Tom Marshall ${ }^{1}$ \\ and Rachel Jordan ${ }^{1}$
}

Affiliations: ${ }^{1}$ Institute of Applied Health Research, College of Medical and Dental Sciences, University of Birmingham, Birmingham, UK. ${ }^{2}$ Dept of Clinical Pharmacy and Toxicology, Maastricht University Medical Centre+ (MUMC+), Maastricht, The Netherlands. ${ }^{3} \mathrm{CIRO}+$, Horn, The Netherlands. ${ }^{4}$ Cardiovascular Research Institute Maastricht (CARIM), Maastricht University, Maastricht, The Netherlands. ${ }^{5}$ Division of Pharmacoepidemiology and Clinical Pharmacology, Utrecht Institute for Pharmaceutical Sciences, Utrecht University, Utrecht, The Netherlands. ${ }^{6}$ Dept of Respiratory Medicine, Maastricht University Medical Centre+ (MUMC+), Maastricht, The Netherlands.

Correspondence: Peymane Adab, Institute of Applied Health Research, College of Medical and Dental Sciences, University of Birmingham, Edgbaston, Birmingham, B15 2TT, UK. E-mail: p.adababham.ac.uk

ABSTRACT The ADO (age, dyspnoea, airflow obstruction) score predicts 3-year overall mortality among chronic obstructive pulmonary disease (COPD) patients. Information on the changes in COPD prognostic scores is sparse and it is unclear if the ADO score should be measured serially.

We followed 4804 UK COPD patients with three or more ADO measurements from The Health Improvement Network (2005-2014) in a retrospective open cohort design. Patient's ADO scores were calculated once per year unless an obstruction or dyspnoea measurement was missing. Cox regression models assessed the independent role of serial ADO scores on mortality. The associations between baseline patient characteristics and long-term change in ADO scores were assessed using linear mixed effect models.

Fewer than $7 \%$ of patients had worsened (i.e. increased) by $\geqslant 1$ point per year after a median follow-up of 4.4 years. There was strong evidence that patients with more rapid worsening in ADO scores had increased mortality (hazard ratio 2.00 (95\% CI 1.59-2.52) per 1 point increase in ADO per year). More rapid ADO score worsening was seen among current smokers (rate difference 0.059 (95\% CI 0.031-0.087); $\mathrm{p}=0.001)$ and ex-smokers (0.028 (95\% CI 0.003-0.054); $\mathrm{p}=0.032)$ and patients with depression $(0.038(95 \%$ CI 0.005-0.071); $\mathrm{p}=0.022)$, while overweight $(-0.0347$ (95\% CI $-0.0544--0.0150) ; \mathrm{p}=0.001)$ and obese $(-0.0412(95 \%$ CI $-0.0625--0.0198) ; \mathrm{p}<0.001)$ patients had a less rapid ADO score worsening.

Serial assessment of the ADO score can identify patients with worsening disease and update their prognosis, especially for patients who smoke, are depressed or have lower body mass index.

@ERSpublications

It is unclear if the ADO score should be measured serially in COPD patients. Serial measurement of the ADO score provides additional information about prognosis in COPD, especially for patients who are smokers, depressed or have lower BMI. http://bit.ly/37A4GUX

Cite this article as: Keene SJ, Adab P, de Vries F, et al. The stability of the ADO score among UK COPD patients from The Health Improvement Network. ERJ Open Res 2020; 6: 00196-2019 [https://doi.org/10.1183/23120541.00196-2019].

This article has supplementary material available from openres.ersjournals.com.

Stata code used for data manipulation and analyses can be provided upon request. THIN Read codes for cases of COPD, covariates and end-points are available upon request.

Received: 7 Aug 2019 | Accepted after revision: 27 Nov 2019

Copyright $\odot$ ERS 2020. This article is open access and distributed under the terms of the Creative Commons Attribution Non-Commercial Licence 4.0. 


\section{Introduction}

Chronic obstructive pulmonary disease (COPD) is a progressive respiratory disease confirmed by the presence of respiratory symptoms in combination with nonreversible airflow limitation [1]. Disease progression is not uniform for all patients and "rapid decliners" have been defined as those with an accelerated decrease in forced expiratory volume in $1 \mathrm{~s}\left(\mathrm{FEV}_{1}\right)$ [2-5]. However, it is now recognised that other components of COPD contribute to its worsening [2,6-8]. Multicomponent prognostic scores can better evaluate the risk of deterioration or death compared with $\mathrm{FEV}_{1}$ alone as they combine multiple domains of COPD. The ADO (age, dyspnoea, airflow obstruction) score combines three easily accessible components and accurately predicts 3 -year mortality $[9,10]$.

However, it is unclear whether or not the ADO score should only be measured at a single point in time [11]. The ADO score may change differently with certain patient characteristics. Deterioration or treatment response may also alter its rate of change and these changes may be predictive of survival. Therefore, it may be important to review and revise mortality predictions in order to better guide management. Information on the changes in prognostic scores for COPD is sparse and no studies have examined the serial measurement of prognostic scores in primary care.

We sought to determine if it is useful to measure the ADO score serially in primary care COPD patients. Our objectives were to examine 1) how serial ADO scores change over time, 2) whether this change was prognostically relevant and 3) which characteristics are related to the rate of change in ADO scores.

\section{Methods}

\section{Study design}

This was a register-based retrospective open cohort study conducted according to the Reporting of Studies Conducted Using Observational Routinely-Collected Health Data (RECORD) statement [12].

\section{Data source}

The Health Improvement Network (THIN) is a longitudinal, clinical primary care database that contains anonymised and validated data on diagnoses, symptoms, hospital referrals, discharge summaries, lifestyle, mortality, prescribing, and clinical and laboratory tests captured by general practitioners using Vision medical software (Vision, London, UK). THIN covers 6\% of the UK population [13].

\section{Study population}

Patients from THIN were included in the study population if they had a current recorded COPD diagnosis Read code assigned by the general practitioner, on (i.e. previously diagnosed patients) or after (i.e. newly diagnosed patients) April 1, 2005. In addition, patients were only included if they had been registered with the practice by April 1, 2004 (i.e. patients moving into the practice at later time-points were excluded), and were alive and contributing data for at least 1 day after April 1, 2005. This date was chosen because it represents 1 year after the introduction of the Quality and Outcomes Framework [14]. In order to accurately estimate the change in ADO score over time, patients were only included in the sample if a minimum of three ADO scores were available (either consecutive or nonconsecutive years). Patients $<40$ years of age in the year of their COPD diagnosis were also excluded as they comprise a minority of the COPD population and are more likely to have a different disease trajectory due to primary asthma or a genetic predisposition such as $\alpha_{1}$-antitrypsin deficiency. Study entry was designated as the date of the baseline ADO score. Their end date was the earliest of the date of death, the date the patient left the practice, the last practice collection date or April 1, 2014.

\section{Patient characteristics}

We obtained sociodemographic data for each participant including sex and Townsend deprivation quintile based on their home postcode (0 to 5 (most deprived); last value recorded). The latest recorded status at any time before study entry was used to define body mass index (BMI) categories (underweight $\left(<18.5 \mathrm{~kg} \cdot \mathrm{m}^{-2}\right)$, normal $\left(18.5-<25 \mathrm{~kg} \cdot \mathrm{m}^{-2}\right)$, overweight $\left(25-<30 \mathrm{~kg} \cdot \mathrm{m}^{-2}\right)$ and obese $\left.\left(\geqslant 30 \mathrm{~kg} \cdot \mathrm{m}^{-2}\right)\right)$ and smoking status (never-smoker, ex-smoker and current smoker). Comorbidities such as ischaemic heart disease, asthma [15], diabetes, heart failure and vascular disease (including transient ischaemic attack, stroke or peripheral arterial disease) were noted as present if there was a relevant clinical code at any time before study entry. Similar to previous studies, a clinical code within the previous 3 years of study entry was used to determine the presence of anxiety and depression [16, 17]. Treatments for COPD were reported present if there was a relevant record of prescription 1 year prior to study entry. Data on the following treatments were available: referral to pulmonary rehabilitation, long-acting muscarinic antagonist (LAMA) prescription (tiotropium), short-acting muscarinic antagonist (SAMA) prescription (ipratropium), long-acting $\beta_{2}$-agonist (LABA) prescription (consisting of salmeterol, formoterol or indacaterol), short-acting $\beta_{2}$-agonist (SABA) prescription (salbutamol or terbutaline) and inhaled 
corticosteroid (ICS) (consisting of budesonide, fluticasone and beclomethasone)-containing prescription (ICS only, ICS+LAMA, ICS+LABA or ICS+LAMA+LABA).

\section{Serial ADO scores}

The overall ADO score is composed of scores assigned to levels for each of its three components: age, dyspnoea (modified Medical Research Council (mMRC) scale) and airflow obstruction (FEV $\%_{1}$ pred). Points were assigned according to the updated ADO publication (supplementary table S1). The study period (2005-2014) was broken up into intervals lasting from April 1 to March 31 in order to reflect years of data capture from routine primary care records. In routine practice, ADO score components are recorded sporadically. Therefore, we calculated the score once per interval, choosing the latest available values for each component in each interval. If either the $\mathrm{FEV}_{1}$ or $\mathrm{mMRC}$ component was not recorded in a certain interval, the score was missing for that interval. The date of each calculable ADO score was designated as the latest date of the $\mathrm{mMRC}$ or $\mathrm{FEV}_{1}$ components in each interval. The rules used to convert raw $\mathrm{FEV}_{1}$ measurements to $\mathrm{FEV}_{1} \%$ pred are provided in the supplementary material. Baseline ADO score and number of calculable ADO scores per patient were added as covariates since both factors could be associated with the rate of change in ADO scores.

\section{Statistical analysis}

Simple linear regression was used to assign rates of change in ADO scores over time to patients. We defined a stable ADO score as a change between -0.5 and +0.5 points per year. Patients with ADO score changes above and below this range were defined as worsening (i.e. increasing) and improving (i.e. decreasing) $\mathrm{ADO}$ score patients, respectively. We then compared baseline characteristics across these groups. Multivariable Cox regression models were used to calculate the hazard ratio for mortality after the date of the final ADO score. Here, we used each individual's change in ADO score over time as a continuous variable and adjusted for the following covariates: ADO score, age, dyspnoea, obstruction, number of ADO measurements, sex, BMI, smoking and selected comorbidities. These covariates were agreed upon by the research team, supported by clinical evidence from the literature. A secondary analysis examined the same association using the aforementioned ADO score change groups as the variable of interest. Finally, using all ADO scores for each participant as the outcome, we built linear mixed effect models to investigate the effect of baseline characteristics on the change in ADO scores over time. Each model was fitted with a random intercept and a random time slope for each patient to account for clustering due to repeated measurements, and contained the following independent variables: time, the characteristic of interest, and an interaction term of the characteristic of interest and time (characteristicxtime), adjusted for covariates listed for the Cox model. Multiple imputation was not used to impute missing ADO scores because mixed effect models are unaffected by complete-case bias [18]. Stata version 14 (StataCorp, College Station, TX, USA) was used for all analyses.

\section{Ethics}

The NHS South East Multi-centre Research Ethics Committee (MREC) approved THIN data collection for research in 2003 subject to independent scientific review, which we obtained (approval 16THIN039) on May 23, 2016.

\section{Results}

Flow of THIN patients into final sample

We identified 67066 COPD patients; 1542 were excluded because they were diagnosed prior to 40 years of age. Of the remaining 65524, a further 60720 did not have at least three calculable ADO scores, leaving 4804 patients with a median (interquartile range) follow-up of 4.38 (3.75-5.55) years. Over half of all those identified with COPD did not have data to derive an ADO score at any time, but around one-third of all calculable ADO measurements were included in the final analysis (table 1). Supplementary table S2 shows comparisons between included (three or more ADO measurements) and excluded patients (less than three ADO measurements).

\section{Description of the rate of change in ADO score}

The mean \pm SD baseline ADO score was $7.4 \pm 2.1$ (range 0-14). The ADO score increased by an average of 0.187 (95\% CI 0.174-0.200) points per year (average number of measurements per patient 3.4). The age component increased by 0.152 (95\% CI $0.149-0.155)$ points, the dyspnoea score increased score by 0.055 (95\% CI 0.050-0.060) points (average number of measurements per patient 5.2) and the obstruction score decreased by 0.009 (95\% CI $0.001-0.016)$ points (average number of measurements per patient 4.3 ) per year (data not shown). The rate of change per patient was approximately normally distributed and 323 (6.7\%) patients had an increase of at least 1 point per year (figure 1). 
TABLE 1 Frequency of calculable ADO (age, dyspnoea, airflow obstruction) score measurements in the overall population of The Health Improvement Network patients

\begin{tabular}{lcc} 
Measurements per subject & Subjects & Total measurements \\
\hline $\mathbf{0}^{\#}$ & $34706(53)$ & $0(0)$ \\
$\mathbf{1}^{\#}$ & $17396(27)$ & $17396(34)$ \\
$\mathbf{2}^{\#}$ & $8618(13)$ & $17236(34)$ \\
$\mathbf{3}$ & $3393(5)$ & $10179(20)$ \\
$\mathbf{4}$ & $1073(2)$ & $4292(8)$ \\
$\mathbf{5}$ & $263(0)$ & $1315(3)$ \\
$\mathbf{6}$ & $59(0)$ & $354(1)$ \\
$\mathbf{7}$ & $13(0)$ & $91(0)$ \\
$\mathbf{8}$ & $3(0)$ & $24(0)$ \\
Total & 65524 & 50877
\end{tabular}

Data are presented as $\mathrm{n}(\%)$; right column calculated by multiplying number of measurements by number of subjects in the same row (middle column). " : subjects were excluded from analysis.

\section{Differences between ADO score change categories}

Using \pm 0.5 points to indicate worsening/improvement, 3766 (78\%) of the included patients had a stable ADO score, whereas $850(18 \%)$ had a worsening ADO score over time and $188(4 \%)$ had an improving ADO score over time. Those with improving ADO scores had fewer ADO measurements (17\% with four or more ADO measurements) than the worsening (27\%) and stable (30\%) groups (table 2). Patients with a worsening $\mathrm{ADO}$ score had the lowest baseline $\mathrm{ADO}$ score (6.6 \pm 2.0 points) and least severe obstruction $\left(\mathrm{FEV}_{1} \%\right.$ pred $64.9 \pm 21.3 \%$ ) and dyspnoea (mMRC score 1.23 \pm 0.97 ). From improving to worsening groups, there was a trend toward more current smokers and normal weight patients and fewer never-smokers and obese patients.

Prognostic role of the change in ADO scores over time

There were $388(8.1 \%)$ deaths in the follow-up period. There was strong evidence $(\mathrm{p}<0.001)$ of a $2.00(95 \%$ CI 1.59-2.52)-fold increase in the rate of mortality per 1 point increase in individual ADO score per year, after adjusting for selected covariates (table 3). Similarly, the association with mortality was stronger in patients grouped in worsening (adjusted HR 2.08 (95\% CI 1.61-2.69)) and improving (adjusted HR 0.49 (95\% CI 0.27-0.91)) categories compared with those with stable scores (reference group) (data not shown).

\section{Characteristics associated with the change in ADO score over time}

Table 4 shows multivariable mixed effect models of the characteristics associated with the change in ADO scores over time. After adjustment for baseline covariates, greater deprivation, recent depression, and prior LABA, LAMA and ICS-containing prescription were all associated with a statistically significant worsening $(\mathrm{p}<0.05)$ of ADO scores over time. Compared with never-smokers, current smokers had a 0.059 (95\% CI 0.031-0.087) points per year worsening of ADO scores. Finally, compared with those with a normal BMI,

FIGURE 1 Histogram of distribution of change in ADO lage, dyspnoea, airflow obstruction) score per year in 4804 included patients.

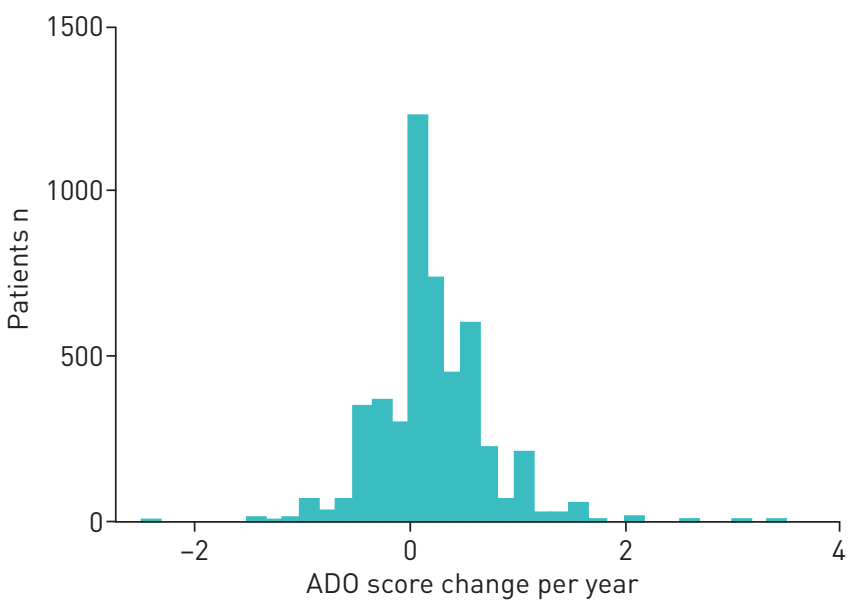




\begin{tabular}{|c|c|c|c|}
\hline & $\begin{array}{l}\text { Improving } \\
\text { ADO change }\end{array}$ & $\begin{array}{c}\text { Stable } \\
\text { ADO change }\end{array}$ & $\begin{array}{l}\text { Worsening } \\
\text { ADO change }\end{array}$ \\
\hline Subjects & 188 & 3766 & 850 \\
\hline Age years & $69.6 \pm 9.5$ & $68.8 \pm 9.2$ & $69.2 \pm 9.5$ \\
\hline Dyspnoea (mMRC score) & $1.44(0.97)$ & $1.94(1.04)$ & $1.23(0.97)$ \\
\hline 0 & $9(4.8)$ & $613(16.3)$ & 237 (27.9) \\
\hline 1 & 71 (37.8) & $1547(41.1)$ & 265 (31.2) \\
\hline 2 & 40 (21.3) & $1016(27.0)$ & $267(31.4)$ \\
\hline 3 & 58 (30.9) & $533(14.2)$ & $75(8.8)$ \\
\hline 4 & $10(5.3)$ & $57(1.5)$ & $6(0.7)$ \\
\hline $\mathrm{FEV}_{1} \%$ pred & $47.6 \pm 15.0$ & $58.6 \pm 19.1$ & $64.9 \pm 21.3$ \\
\hline First ADO score & $8.9 \pm 1.9$ & $7.6 \pm 2.0$ & $6.6 \pm 2.0$ \\
\hline $0-5$ & $7(3.7)$ & $543(14.4)$ & $239(28.1)$ \\
\hline 6 or 7 & $33(17.6)$ & 1262 (33.5) & 333 (39.2) \\
\hline 8 or 9 & 77 (41.0) & 1355 (36.0) & $230(27.1)$ \\
\hline $10-14$ & 71 (37.8) & $606(16.1)$ & 48 (5.7) \\
\hline Four or more ADO measurements & $32(17.0)$ & $1146(30.4)$ & $233(27.4)$ \\
\hline Female & $96(51.1)$ & 1676 (44.5) & $379(44.6)$ \\
\hline White ethnicity & 99 (100.0) & $1848(98.4)$ & $426(98.2)$ \\
\hline \multicolumn{4}{|l|}{ Townsend deprivation quintile } \\
\hline 1 (least deprived) & $27(14.6)$ & $643(17.4)$ & 145 (17.3) \\
\hline 2 & $37(20.0)$ & $692(18.8)$ & $151(18.0)$ \\
\hline 3 & $41(22.2)$ & 805 (21.8) & $186(22.2)$ \\
\hline 4 & $43(23.2)$ & $820(22.3)$ & 195 (23.3) \\
\hline 5 (most deprived) & $37(20.0)$ & $726(19.7)$ & $161(19.2)$ \\
\hline \multicolumn{4}{|l|}{ Smoking status } \\
\hline Current smoker & $49(26.9)$ & $994(28.1)$ & 258 (32.0) \\
\hline Ex-smoker & $104(57.1)$ & $2066(58.4)$ & $468(58.0)$ \\
\hline Never-smoker & $29(15.9)$ & 478 (13.5) & $81(10.0)$ \\
\hline BMI $\mathrm{kg} \cdot \mathrm{m}^{-2}$ & $28.3 \pm 6.3$ & $27.5 \pm 5.5$ & $26.9 \pm 5.6$ \\
\hline \multicolumn{4}{|l|}{ BMI category } \\
\hline Underweight & 7 (3.9) & 90 (2.5) & 27 (3.3) \\
\hline Normal & 50 (27.9) & $1130(31.8)$ & $300(36.9)$ \\
\hline Overweight & $70(39.1)$ & 1324 (37.3) & $281(34.5)$ \\
\hline Obese & $52(29.1)$ & 1005 (28.3) & 206 (25.3) \\
\hline LAMA prescription & $66(35.1)$ & $1000(26.6)$ & 266 (31.3) \\
\hline LABA prescription & $53(28.2)$ & 1165 (30.9) & 259 (30.5) \\
\hline SAMA prescription & 31 (16.5) & $603(16.0)$ & $143(16.8)$ \\
\hline SABA prescription & $118(62.8)$ & $2212(58.7)$ & $518(60.9)$ \\
\hline ICS-containing prescription & $52(27.7)$ & 1306 (34.7) & $287(33.8)$ \\
\hline Pulmonary rehabilitation referral & 6 (3.2) & $176(4.7)$ & $29(3.4)$ \\
\hline Heart failure & $11(5.9)$ & $192(5.1)$ & $42(4.9)$ \\
\hline Ischaemic heart disease & 37 (19.7) & $716(19.0)$ & $181(21.3)$ \\
\hline Anxiety & 7 (3.7) & $86(2.3)$ & $16(1.9)$ \\
\hline Depression & $12(6.4)$ & $238(6.3)$ & $55(6.5)$ \\
\hline Diabetes & $28(14.9)$ & 469 (12.5) & 96 (11.3) \\
\hline TIA, stroke or PAD & 25 (13.3) & 443 (11.8) & $121(14.2)$ \\
\hline Asthma & 68 (36.2) & 1351 (35.9) & $311(36.6)$ \\
\hline
\end{tabular}

overweight $(\beta=-0.035 \quad(95 \% \quad \mathrm{CI} \quad-0.0544--0.0150) ; \mathrm{p}=0.001)$ and obese $(\beta=-0.041 \quad(95 \%$ CI $-0.0625-0.0198) ; \mathrm{p}<0.001)$ patients showed improvement over time and underweight patients had a worsening ADO score of 0.041 (95\% CI -0.018-0.100) points per year. 


\begin{tabular}{|c|c|c|}
\hline & $\operatorname{HR}(95 \% \mathrm{CI})$ & p-value \\
\hline Change in ADO score over time (per 1 point increase per year) & $2.00(1.59-2.52)$ & $<0.001$ \\
\hline Baseline ADO Score (per 1 point increase) & $1.28(1.10-1.50)$ & 0.002 \\
\hline Age at baseline (per 1 year increase) & $1.03(1.00-1.05)$ & 0.074 \\
\hline mMRC at baseline (per 1 point increase) & $1.18(1.03-1.36)$ & 0.017 \\
\hline $\mathrm{FEV}_{1} \%$ pred (per 1 percentage point increase) & $0.99(0.98-1.01)$ & 0.277 \\
\hline Number of ADO measurements (per measurement) & $0.79(0.65-0.95)$ & 0.010 \\
\hline Female sex & $0.88(0.71-1.10)$ & 0.262 \\
\hline \multicolumn{3}{|l|}{ BMI category" } \\
\hline Underweight & $1.71(1.16-2.51)$ & 0.006 \\
\hline Normal & Reference & \\
\hline Overweight & $0.63(0.49-0.80)$ & $<0.001$ \\
\hline Obese & $0.62(0.47-0.83)$ & $<0.001$ \\
\hline \multicolumn{3}{|l|}{ Smoking status ${ }^{\#}$} \\
\hline Never-smoker & Reference & \\
\hline Ex-smoker & $1.08(0.79-1.48)$ & 0.626 \\
\hline Current smoker & $1.27(0.87-1.83)$ & 0.148 \\
\hline Presence of heart failure ${ }^{\#}$ & $1.60(1.19-2.14)$ & 0.002 \\
\hline Presence of ischaemic heart disease ${ }^{\#}$ & $1.26(1.00-1.58)$ & 0.054 \\
\hline Presence of diabetes mellitus ${ }^{\#}$ & $0.98(0.74-1.30)$ & 0.873 \\
\hline Presence of TIA, stroke or PAD" & $1.24(0.97-1.58)$ & 0.092 \\
\hline Presence of asthma ${ }^{\#}$ & $1.01(0.82-1.26)$ & 0.898 \\
\hline \multicolumn{3}{|c|}{$\begin{array}{l}\text { mMRC: modified Medical Research Council; } \mathrm{FEV}_{1} \text { : forced expiratory volume in } 1 \mathrm{~s} ; \mathrm{BMI} \text { : body mass index; } \\
\text { TIA: transient ischaemic attack; PAD: peripheral arterial disease. The proportional hazards assumption for } \\
\text { serial ADO scores was not violated ( } p=0.7214) \text {. The median (interquartile range) time between the first and } \\
\text { final ADO score was } 3.54(2.71-4.45) \text { years. \#: most recent status prior to the last ADO score } \\
\text { measurement. }\end{array}$} \\
\hline
\end{tabular}

\section{Discussion}

This retrospective longitudinal study showed that most COPD primary care patients had stable disease over a median follow-up of $>4$ years. However, serial ADO scores had prognostic value beyond the initial measurement. Thus, serial assessment of the ADO score may be needed in order to update the predicted risk of death. We found that this may be particularly important for patients with lower BMI, depression and those who are current or ex-smokers.

In contrast to our study, a prospective study of COPD patients followed for a period of 3 years following hospital admission with an acute exacerbation found that the baseline BODE (BMI, obstruction, dyspnoea, exercise capacity) score, but not changes in the BODE score, predicted survival [19]. The authors concluded that a single measurement, rather than serial measurements, of the BODE score would be sufficient for prognostication [19]. However, two other studies showed that before and after lung volume reduction surgery, changes in the BODE score and the final BODE score (patients tended to improve with surgery) were independently associated with mortality in severe emphysema patients [20, 21]. Similarly, pulmonary rehabilitation improved the BODE score and its change added prognostic information for 246 COPD outpatients in the USA [22]. Combined, serial BODE measurements may be more helpful in assessing treatment response rather than disease worsening.

It is well known that low BMI is associated with increased risk of mortality in COPD patients [23]. We found that lower BMI was associated with worsening disease. Similarly, COPD secondary care patients in the BODE cohort were more likely to have worsening obstruction with low BMI than with normal BMI at baseline [2]. While BMI may be associated with disease worsening, obese patients may have trouble breathing due to their weight, resulting in overdiagnosis of COPD [24] and more stable ADO scores over time in our study. Second, the effect of smoking on longitudinal lung function deterioration has long been documented [4]. A secondary analysis of the Lung Health Study randomised controlled trial showed that there was a greater decline in lung function over 11 years if participants were continuous smokers $\left(60 \mathrm{~mL} \cdot y_{e a r}{ }^{-1}\right)$ compared with intermittent quitters $\left(48 \mathrm{~mL} \cdot \mathrm{year}^{-1}\right)$ and sustained quitters $\left(27 \mathrm{~mL} \cdot \mathrm{year}^{-1}\right)$ [25]. Although reducing smoking can improve decline in $\operatorname{FEV}_{1}[26,27]$, nearly complete cessation may be necessary for demonstrable benefit [28]. Next, it may be difficult to diagnose depression in COPD patients 


\begin{tabular}{|c|c|c|}
\hline Characteristics interacting with time & $\beta(95 \% \mathrm{CI})$ & p-value \\
\hline Baseline ADO score & $-0.0397(-0.0437--0.0357)$ & $<0.001$ \\
\hline Number of ADO measurements & $0.0178(0.0080-0.0276)$ & $<0.001$ \\
\hline Age at baseline years & $-0.0001(-0.0010-0.0008)$ & 0.885 \\
\hline mMRC score at baseline & $-0.0446(-0.0530--0.0362)$ & $<0.001$ \\
\hline $\mathrm{FEV}_{1} \%$ pred at baseline & $0.0026(0.0022-0.0031)$ & $<0.001$ \\
\hline Townsend quintile & $0.0062(0.0001-0.0123)$ & 0.045 \\
\hline Female sex & $0.0001(-0.0164-0.0168)$ & 0.982 \\
\hline Heart failure (any time) & $-0.0044(-0.0413-0.0327)$ & 0.817 \\
\hline Ischaemic heart disease (any time) & $-0.0001(-0.0211-0.0208)$ & 0.989 \\
\hline Asthma (any time) & $-0.0035(-0.0206-0.0136)$ & 0.688 \\
\hline Anxiety ( 3 years prior) & $-0.0191(-0.0770-0.0389)$ & 0.519 \\
\hline Depression ( 3 years prior) & $0.0384(0.0054-0.0713)$ & 0.022 \\
\hline Diabetes (any time) & $-0.0084(-0.0346-0.0178)$ & 0.531 \\
\hline TIA, stroke or PAD (any time) & $-0.0035(-0.0288-0.0217)$ & 0.783 \\
\hline LAMA prescription (1 year prior) & $0.0236(0.0045-0.0427)$ & 0.016 \\
\hline LABA prescription (1 year prior) & $0.0186(0.0008-0.0365)$ & 0.041 \\
\hline SAMA prescription (1 year prior) & $0.0195(-0.0019-0.0409)$ & 0.075 \\
\hline SABA prescription (1 year prior) & $0.0137(-0.0031-0.0306)$ & 0.111 \\
\hline ICS-containing prescription (1 year prior) & $0.0189(0.0017-0.0361)$ & 0.031 \\
\hline Pulmonary rehabilitation referral (1 year prior) & $0.0029(-0.0372-0.0430)$ & 0.886 \\
\hline \multicolumn{3}{|l|}{ BMI category (most recent status) } \\
\hline Underweight & $0.0411(-0.0175-0.0996)$ & 0.169 \\
\hline Normal & Reference & \\
\hline Overweight & $-0.0347(-0.0544--0.0150)$ & 0.001 \\
\hline Obese & $-0.0412(-0.0625--0.0198)$ & $<0.001$ \\
\hline \multicolumn{3}{|l|}{ Smoking status (most recent status) } \\
\hline Never-smoker & Reference & \\
\hline Ex-smoker & $0.0282(0.0025-0.0539)$ & 0.032 \\
\hline Current smoker & $0.0588(0.0311-0.0866)$ & $<0.001$ \\
\hline \multicolumn{3}{|c|}{$\begin{array}{l}\text { mMRC: modified Medical Research Council; FEV }{ }_{1} \text { : forced expiratory volume in } 1 \mathrm{~s} ; \text { TIA: transient } \\
\text { ischaemic attack; PAD: peripheral artery disease; LAMA: long-acting muscarinic antagonist; LABA: } \\
\text { long-acting } \beta_{2} \text {-agonist; SAMA: short-acting muscarinic antagonist; SABA: short-acting } \beta_{2} \text {-agonist; ICS: } \\
\text { inhaled corticosteroid; BMI: body mass index. }\end{array}$} \\
\hline
\end{tabular}

because of overlapping symptoms [29]. Patients in our study with worsening COPD may be more likely to be depressed if respiratory symptoms are limiting their social lives [30]. Finally, we found that disease worsening was greater in those who had received respiratory pharmacotherapy. Similar to depression, these findings may be due to reverse causation, reflecting that those on a worsening disease trajectory had been started on pharmacotherapy.

The current study has several strengths and limitations. First, previous studies have used longitudinal lung function measurements alone to describe COPD progression. However, COPD is a heterogeneous disease and patients may worsen despite stable lung function [2]. A multicomponent prognostic score more accurately accounts for disease heterogeneity. It also allows changes in the score to be placed into the context of changes in individual risk of mortality. Next, unlike previous studies that examined serial measurements of prognostic scores, we included primary care patients, where COPD is mainly managed [31]. However, despite a large sample size, we excluded many patients due to the limited availability of data. These patients were different in a few characteristics compared with the whole population and may have had more stable disease, requiring fewer dyspnoea and obstruction measurements from their general practitioner. Although THIN is generalisable to the UK for demographics, disease prevalence and mortality rates [13], patients from urban areas may be over-represented because Vision software use is clustered in these areas [32]. Next, unmeasured confounding and unstandardised measurements were unavoidable. The latter may partly explain the improvement in average $\mathrm{FEV}_{1} \%$ pred over time in our sample. Additionally, $\mathrm{FEV}_{1} \%$ pred may be flawed when examining its change over time. $\mathrm{FEV}_{1} \%$ pred would increase if a patient ages (and/or becomes shorter) despite relatively stable $\mathrm{FEV}_{1}$ (in litres). 
Longitudinal ADO score trends were assumed to be linear to ease interpretation, but this may not have been true for some patients. Finally, the ADO score provides an estimate of risk that can be used to support clinical discussions with patients and joint decision making. Serial measurement may refine risk estimates and identify those who have a worsening disease trajectory, but we do not yet have evidence whether stratified management, informed by risk scores, would modify patient outcomes.

\section{Conclusions}

Given the wide range of clinical courses in patients with COPD, it is important to understand whether and how prognostic scores change over time in order to identify patients with worsening disease. If this change has prognostic relevance or is related to patient characteristics, then serial assessment may be useful. One-time use of the ADO score could help define treatment options that could be weighed against the current risk of mortality [9]. However, serial assessment of the ADO score can identify patients with worsening disease and update their prognosis, especially for patients who smoke, are depressed or have lower BMI.

Acknowledgements: Results from the study were presented as an oral presentation at the European Respiratory Society International Congress in September, 2019 (Madrid, Spain).

Author contributions: P. Adab takes full responsibility for the data, content of the manuscript and analysis. P. Adab and R. Jordan conceptualised the study. S.J. Keene undertook the statistical analysis with advice, direction and guidance from J. Martin and A. Sitch. S.J. Keene wrote the first draft of the paper. T. Marshall supplied the dataset and gave advice on THIN data. F.M.E. Franssen and F. de Vries reviewed all drafts and gave advice on study direction. All authors reviewed and approved the final submission.

Conflict of interest: S.J. Keene has nothing to disclose. P. Adab reports an NIHR Programme Grant during the conduct of the study, and grants from the NIHR outside the submitted work. P. Adab is deputy chair of the NIHR PHR funding committee. F. de Vries supervises two PhD students who are employed by F. Hoffmann-La-Roche Ltd (Basel, Switzerland and Welwyn Garden City, UK). The topics of their PhDs do not relate to the current study and F. de Vries has not received any fees or reimbursements for this. F.M.E. Franssen reports personal fees from AstraZeneca, Boehringer Ingelheim, Chiesi, GlaxoSmithKline, Novartis and Teva, outside the submitted work. A. Sitch reports an NIHR Programme Grant during the conduct of the study, and a grant for an investigator-initiated study from AstraZeneca outside the submitted work. J. Martin has nothing to disclose. T. Marshall has nothing to disclose. R. Jordan holds a grant from NIHR that supported the cohort used in the submitted manuscript.

\section{References}

1 Mannino DM, Buist AS. Global burden of COPD: risk factors, prevalence, and future trends. Lancet 2007; 370: 765-773.

2 Casanova C, De Torres JP, Aguirre-Jaíme A, et al. The progression of chronic obstructive pulmonary disease is heterogeneous: the experience of the BODE cohort. Am J Respir Crit Care Med 2011; 184: 1015-1021.

3 de-Torres JP, Marín JM, Pinto-Plata V, et al. Is COPD a progressive disease? A long term BODE cohort observation. PLoS One 2016; 11: e0151856.

4 Fletcher C, Peto R. The natural history of chronic airflow obstruction. Br Med J 1977; 1: 1645-1648.

5 Miller A, Raskin JM. The natural history of COPD: confirming and going beyond Fletcher and Peto. Eur Respir J 2014; 44: 280-283.

6 Wedzicha JA. The heterogeneity of chronic obstructive pulmonary disease. Thorax 2000; 55: 631-632.

7 Rennard SI, Drummond MB. Early chronic obstructive pulmonary disease: definition, assessment, and prevention. Lancet 2015; 385: 1778-1788.

8 Rennard SI, Vestbo J. Natural histories of chronic obstructive pulmonary disease. Proc Am Thorac Soc 2008; 5 878-883.

9 Puhan MA, Hansel NN, Sobradillo P, et al. Large-scale international validation of the ADO index in subjects with COPD: an individual subject data analysis of 10 cohorts. BMJ Open 2012; 2: e002152.

10 Guerra B, Haile SR, Lamprecht B, et al. Large-scale external validation and comparison of prognostic models: an application to chronic obstructive pulmonary disease. BMC Med 2018; 16: 33.

11 Miravitlles M, Vogelmeier C, Roche N, et al. A review of national guidelines for management of COPD in Europe. Eur Respir J 2016; 47: 625-637.

12 Benchimol EI, Smeeth L, Guttmann A, et al. The REporting of studies Conducted using Observational Routinely-collected health Data (RECORD) statement. PLoS Med 2015; 12: e1001885.

13 Blak BT, Thompson M, Dattani H, et al. Generalisability of The Health Improvement Network (THIN) database: demographics, chronic disease prevalence and mortality rates. Inform Prim Care 2011; 19: 251-255.

14 Smith CJP, Gribbin J, Challen KB, et al. The impact of the 2004 NICE guideline and 2003 General Medical Services contract on COPD in primary care in the UK. QJM 2008; 101: 145-153.

15 Battaglia S, Benfante A, Spatafora M, et al. Asthma in the elderly: a different disease? Breathe 2016; 12: 18-28.

16 Haroon S, Adab P, Riley RD, et al. Predicting risk of COPD in primary care: development and validation of a clinical risk score. BMJ Open Respir Res 2014; 1: e000060.

17 Haroon S, Adab P, Riley RD, et al. Predicting risk of undiagnosed COPD: development and validation of the TargetCOPD score. Eur Respir J 2017; 49: 1602191.

18 Ibrahim JG, Molenberghs G. Missing data methods in longitudinal studies: a review. Test 2009; 18: 1-43.

19 Ko FWS, Tam W, Tung AHM, et al. A longitudinal study of serial BODE indices in predicting mortality and readmissions for COPD. Respir Med 2011; 105: 266-273.

20 Martinez FJ, Han MK, Andrei A-C, et al. Longitudinal change in the BODE index predicts mortality in severe emphysema. Am J Respir Crit Care Med 2008; 178: 491-499. 
Imfeld S, Bloch KE, Weder W, et al. The BODE index after lung volume reduction surgery correlates with survival. Chest 2006; 129: 873-878.

22 Cote CG, Celli BR. Pulmonary rehabilitation and the BODE index in COPD. Eur Respir J 2005; 26: 630-636.

23 Yamauchi Y, Hasegawa W, Yasunaga H, et al. Paradoxical association between body mass index and in-hospital mortality in elderly patients with chronic obstructive pulmonary disease in Japan. Int J Chron Obstruct Pulmon Dis 2014; 9: 1337-1346.

24 Verberne LDM, Leemrijse CJ, Swinkels ICS, et al. Overweight in patients with chronic obstructive pulmonary disease needs more attention: a cross-sectional study in general practice. NPJ Prim Care Respir Med 2017; 27 : 63.

25 Anthonisen NR, Connett JE, Murray RP. Smoking and lung function of Lung Health Study participants after 11 years. Am J Respir Crit Care Med 2002; 166: 675-679.

26 Scanlon PD, Connett JE, Waller LA, et al. Smoking cessation and lung function in mild-to-moderate chronic obstructive pulmonary disease: the Lung Health Study. Am J Respir Crit Care Med 2000; 161: 381-390.

27 Murray RP, Anthonisen NR, Connett JE, et al. Effects of multiple attempts to quit smoking and relapses to smoking on pulmonary function. J Clin Epidemiol 1998; 51: 1317-1326.

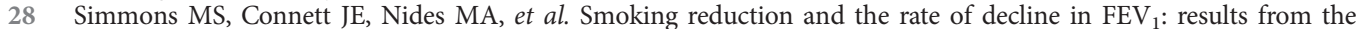
Lung Health Study. Eur Respir J 2005; 25: 1011-1017.

29 Yohannes AM, Alexopoulos GS. Depression and anxiety in patients with COPD. Eur Respir Rev 2014; 23: 345-349.

30 Franssen FME, Smid DE, Deeg DJH, et al. The physical, mental, and social impact of COPD in a population-based sample: results from the Longitudinal Aging Study Amsterdam. NPJ Prim Care Respir Med 2018; $28: 30$

31 James GD, Donaldson GC, Wedzicha JA, et al. Trends in management and outcomes of COPD patients in primary care, 2000-2009: a retrospective cohort study. NPJ Prim Care Respir Med 2014; 24 : 14015.

32 Kontopantelis E, Stevens RJ, Helms PJ, et al. Spatial distribution of clinical computer systems in primary care in England in 2016 and implications for primary care electronic medical record databases: a cross-sectional population study. BMJ Open 2018; 8: e020738. 